\title{
Optimal Isotropic Wavelets for Localized Tight Frame Representations
}

\author{
John Paul Ward, Pedram Pad, and Michael Unser
}

\begin{abstract}
In this letter, we aim to identify the optimal isotropic mother wavelet for a given spatial dimension based on a localization criterion. Within the framework of the calculus of variations, we specify an Euler-Lagrange equation for this problem, and we find the unique analytic solutions. In the one- and two-dimensional cases, the derived wavelets are well known.
\end{abstract}

Index Terms-Calculus of variations, localization, Simoncelli wavelets, wavelet tight frames.

\section{INTRODUCTION}

A N ISOTROPIC multiresolution analysis, as defined in [1], consists of subspaces that are invariant under rotation and translation. These invariances are ideal for image processing tasks, since many natural images contain features that are similar up to a rigid motion.

The property of invariance to Euclidean motions restricts the class of wavelets that we can consider. Essentially, the mother wavelet must be band-limited, cf. [1, Proposition 2.2]. We also require the wavelet to generate a tight frame so that the wavelet system is robust to perturbations and satisfies a Parseval-type energy preservation property [2]. If we further add an assumption on its support in the Fourier domain (cf. Assumption 2), then we can take advantage of a fast filterbank implementation [3, Section IV]. A general construction for wavelets of this type can be found in [4], and commonly used versions include the following: Held [5], Papadakis [1], and Simoncelli [6] (which was given as an example in [4]). An overview of these can be found in [3]. Such wavelets, and their extensions, have numerous applications, e.g. [1], [5]-[10].

In this letter, we consider the optimal wavelet to be the one that is the most localized. Poorly localized wavelets have long range influence on a signal, so small errors are propagated over longer distances. Localization, or concentration in a neighborhood of a point, is important for reducing boundary artifacts. Also, better localization reduces approximation error in computer discritization.

Manuscript received March 27, 2015; revised June 09, 2015; accepted June 17, 2015. Date of publication June 22, 2015; date of current version June 24 2015. This work was supported by the European Research Council under the European Union's Seventh Framework Programme (FP7/2007-2013)/ERC Grant Agreement 267439, and in part by the Swiss National Science Foundation under Grant 200020-144355. The associate editor coordinating the review of this manuscript and approving it for publication was Prof. Glenn Easley.

The authors are with the Biomedical Imaging Group, École polytechnique fédérale de Lausanne (EPFL), CH-1015 Lausanne VD, Switzerland.

Color versions of one or more of the figures in this paper are available online at http://ieeexplore.ieee.org.

Digital Object Identifier 10.1109/LSP.2015.2448233
Variance criteria for finding optimal basis function are common in the literature. One of the earliest works in this direction is [11], where Gabor identifies the modulated Gaussian as the one-dimensional function that provides an equality for the Heisenberg uncertainty principle. His techniques are similar to ours, as he uses the the calculus of variations. Also, the authors of [12] use an uncertainty principle to motivate their construction of polyharmonic B-splines and corresponding wavelets.

Several authors have studied prolate spheriodal wave functions as minimizers of localization criteria [13]-[17]. In [18], the authors consider the variance of radial wavelets; however, they do not impose our tight frame constraint. Furthermore, in [19] and [20], the authors propose localized tight frames on spheres, again using a variance criterion.

The main difference between our work and these previous results is that we directly impose the tight frame constraint and use it as a normalization factor, rather than an $L_{2}$ term. The closest work to ours is [21], where the authors numerically optimized tight frame mother wavelets with an added $L_{2}$ normalization term. They also consider a one-dimensional variance of the wavelet profile, rather than the two-dimensional variance of the wavelet itself. Let us also point out that our localization criterion has a simpler formulation, which allows us to solve the problem analytically.

It is interesting to note that in the one- and two-dimensional setting, we recover known wavelets. The optimal wavelet for the one-dimensional case is in the Held [5] family. In the two-dimensional case, the derived wavelet is the one that was proposed by Simoncelli for his equiangular design [6].

In the remainder of this section, we cover background material on wavelets, tight frames, and radial Fourier transforms. We also describe our localization criteria. In Section II, we precisely formulate the problem that we consider and interpret it in the Fourier domain in a simplified form. In Section III, we present the optimal wavelets as analytic solutions of Euler-Lagrange equations, and we plot some of them.

\section{A. Background and Preliminaries}

The Fourier transform of a function $f \in L_{1}\left(\mathbb{R}^{d}\right)$ is

$$
\mathcal{F} f(\boldsymbol{\omega})=\frac{1}{(2 \pi)^{d / 2}} \int_{\mathbb{R}^{d}} f(\boldsymbol{x}) e^{-i \boldsymbol{x} \cdot \boldsymbol{\omega}} d \boldsymbol{x},
$$

where $\boldsymbol{x} \cdot \boldsymbol{\omega}$ denotes the usual inner product on $\mathbb{R}^{d}$.

For a radial function $\Phi(\boldsymbol{x})=\phi(|\boldsymbol{x}|)$, its Fourier transform is also radial; i.e., $\mathcal{F} \Phi(\boldsymbol{\omega})=\varphi_{d}(|\boldsymbol{\omega}|)$. The one-dimensional 
frequency domain profile $\varphi_{d}$ can be computed directly from the one-dimensional spatial profile as

$$
\varphi_{d}(\rho)=\rho^{-(d-2) / 2} \int_{[0, \infty)} \phi(r) r^{d / 2} J_{(d-2) / 2}(\rho r) \mathrm{d} r
$$

cf. [22, Chapter 5], and we use $\mathcal{H}_{d}$ to denote this Hankel transform mapping; i.e, $\varphi_{d}=\mathcal{H}_{d} \phi$. When the derivatives and Fourier transforms exist, we have the dimension walk result

$$
\varphi_{d+2}(\rho)=\frac{-1}{\rho} \varphi_{d}^{\prime}(\rho)
$$

cf. [23, Theorem 1].

Let $\Phi: \mathbb{R}^{d} \rightarrow \mathbb{R}$ be a radial function. We define its dilated and shifted versions as

$$
\Phi_{j, \boldsymbol{k}}:=2^{j d / 2} \Phi\left(2^{j}(\cdot)-\boldsymbol{k}\right) .
$$

for $j \in \mathbb{Z}$ and $\boldsymbol{k} \in \mathbb{Z}^{d}$. We use the dot notation, so the symbol $(\cdot)$ is a placeholder for the variable $\boldsymbol{x} \in \mathbb{R}^{d}$.

We say that the mother wavelet $\Phi$ generates a tight frame if

$$
\|f\|_{L_{2}}^{2}=\sum_{j, \boldsymbol{k}}\left|\left\langle f, \Phi_{j, \boldsymbol{k}}\right\rangle\right|^{2}
$$

for all $f \in L_{2}\left(\mathbb{R}^{d}\right)$. This implies that the wavelet transformation is self-reversible in the sense that

$$
f=\sum_{j, \boldsymbol{k}}\left\langle f, \Phi_{j, \boldsymbol{k}}\right\rangle \Phi_{j, \boldsymbol{k}}
$$

\section{B. Measure of Localization}

Our aim is to make the representation of any function $f \in$ $L_{2}\left(\mathbb{R}^{d}\right)$ in the wavelet frame as local as possible. The local energy of a function $f$ in a given neighborhood should be captured by the wavelets that are centered close to that neighborhood.

Proposition 1: Suppose $f \in L_{2}\left(\mathbb{R}^{d}\right)$ is supported in the ball of radius $R$ centered at the origin. For a given $n \in \mathbb{N}$, there is a constant $C>0$, depending on $n, j$, and $R$ such that the wavelet coefficients of $f$ satisfy

$$
\left|\left\langle f, \Phi_{j, \boldsymbol{k}}\right\rangle\right| \leq C|\boldsymbol{k}|^{-n}\left\||\cdot|^{n} \Phi_{j, \mathbf{0}}\right\|_{L_{2}}\|f\|_{L_{2}},
$$

for $|\boldsymbol{k}|$ sufficiently large.

Proof: The coefficients are

$$
\left\langle f, \Phi_{j, \boldsymbol{k}}\right\rangle=\int_{\mathbb{R}^{d}} f(\boldsymbol{x}) \Phi_{j, \mathbf{0}}\left(\boldsymbol{x}-2^{-j} \boldsymbol{k}\right) \mathrm{d} \boldsymbol{x} .
$$

Changing variables, we write

$$
\left\langle f, \Phi_{j, \boldsymbol{k}}\right\rangle=\int_{\mathbb{R}^{d}}\left(f\left(\boldsymbol{y}+2^{-j} \boldsymbol{k}\right)|\boldsymbol{y}|^{-n}\right)\left(|\boldsymbol{y}|^{n} \Phi_{j, \mathbf{0}}(\boldsymbol{y})\right) \mathrm{d} \boldsymbol{y} .
$$

Finally, we require $|\boldsymbol{k}|>2^{j} R$ and use the support condition along with the Cauchy-Schwartz inequality to obtain the bound in (7).

In the previous proposition, the number $n$ specifies the rate of decay of the coefficients as $|\boldsymbol{k}| \rightarrow \infty$, while the term $\left\||\cdot|^{n} \Phi_{j, \boldsymbol{k}}\right\|_{L_{2}}$ affects the size of the constant in front.

Also, note that while this proposition is stated for compactly supported functions, there is a similar result for general $L_{2}$ func- tions $f$. One can view such an $f$ as an infinite sum of compactly supported functions $f_{l}$, centered at the lattice points $l \in \mathbb{Z}^{d}$. The function $f_{\boldsymbol{l}}$ contains the local information of $f$ near $\boldsymbol{l}$, and the wavelet coefficients of $f_{l}$ satisfy the estimate

$$
\left|\left\langle f_{\boldsymbol{l}}, \Phi_{j, \boldsymbol{k}}\right\rangle\right| \leq C|\boldsymbol{k}-\boldsymbol{l}|^{-n}\left\||\cdot|^{n} \Phi_{j, \mathbf{0}}\right\|_{L_{2}}\left\|f_{\boldsymbol{l}}\right\|_{L_{2}}
$$

in analogy with (7).

Considering this analysis, we would like to use a wavelet for which $\left\||\cdot|^{n} \Phi_{j, \mathbf{0}}\right\|_{L_{2}}^{2}$ is minimal. Since the weight function $|\cdot|^{n}$ is homogeneous, it suffices to solely consider the case $j=0$. Moreover, the choice $n=1$ corresponds to a non-normalized variance of $\Phi_{j, 0}$, and we shall focus on this case.

In summary, we propose the following measure of localization for an isotropic wavelet $\Phi$ :

$$
V(\Phi):=\int_{\mathbb{R}^{d}}|\Phi(\boldsymbol{x})|^{2}|\boldsymbol{x}|^{2} \mathrm{~d} \boldsymbol{x},
$$

subject to the constraint (5).

\section{Problem Formulation}

Our goal is to identify the isotropic wavelet $\Phi(\boldsymbol{x})=\phi(|\boldsymbol{x}|)$ that minimizes $V(\Phi)$ as defined in (11). We assume that $\Phi$ is the mother wavelet for a tight wavelet frame, and its Fourier transform is the isotropic function $\mathcal{F} \Phi(\boldsymbol{\omega})=\varphi_{d}(|\boldsymbol{\omega}|)$.

Assumption 2: The support of $\varphi_{d}$ is $[\pi / 4, \pi]$.

This assumption on the support of $\varphi_{d}$ means that the tight frame constraint (5) is equivalent to

$$
\sum_{k \in \mathbb{Z}} \varphi_{d}\left(2^{k} \rho\right)^{2}=1
$$

for every $\rho>0,[24$, Chapter 3].

\section{A. Fourier Domain Interpretation}

Using properties of the Fourier transform, we interpret $V(\Phi)$ as a functional on the Fourier profile $\varphi_{d}$. In the following, $\propto$ denotes equality up to a multiplicative constant; this simplifies the expression, without affecting the minimization problem. The Plancherel relationship for Fourier implies

$$
\begin{aligned}
V(\Phi) & \propto \int_{[0, \infty)}|\phi(r)|^{2} r^{d+1} \mathrm{~d} r \\
& \propto \int_{[0, \infty)}\left|\varphi_{d+2}(\rho)\right|^{2} \rho^{d+1} \mathrm{~d} \rho .
\end{aligned}
$$

Using (3), we have

$$
V(\Phi) \propto \int_{[0, \infty)}\left|\varphi_{d}^{\prime}(\rho)\right|^{2} \rho^{d-1} \mathrm{~d} \rho .
$$

Notice that this functional involves a first order differential operator applied to $\varphi_{d}$. Choosing a larger values of $n$ leads to a more complex functional with an $n$th order differential operator applied to $\varphi_{d}$. We postpone the analysis of this higher order case to a future work.

\section{B. Tight Frame Condition}

Here, we include the tight frame condition (12) in (15). Together with the support assumption on $\varphi_{d},(12)$ means that $\varphi_{d}$ 


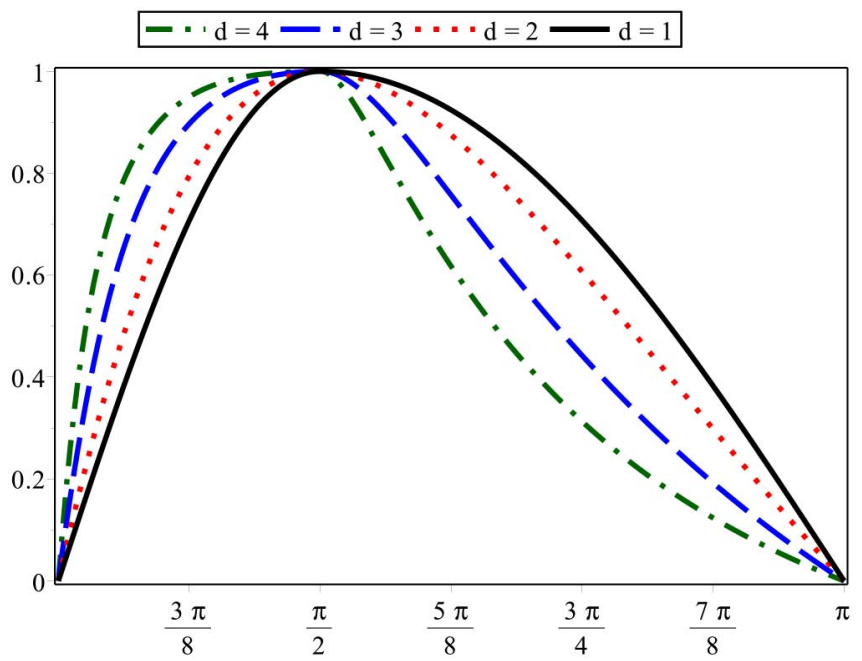

Fig. 1. Plots of the optimal wavelet profiles $\varphi_{d}$ for $d=1,2,3,4$.

is specified by its values on $[\pi / 2, \pi]$, a proper subset of its support. On $[\pi / 4, \pi / 2]$

$$
\varphi_{d}(\rho)=\sqrt{1-\varphi_{d}(2 \rho)^{2}} .
$$

Using this equation, we restate $V(\Phi)$ as an integral on $[\pi / 2, \pi]$.

Taking the derivative of (16),

$$
\varphi_{d}^{\prime}(\rho)=-2 \frac{\varphi_{d}(2 \rho) \varphi_{d}^{\prime}(2 \rho)}{\sqrt{1-\varphi_{d}(2 \rho)^{2}}} .
$$

A change of variables gives

$$
\begin{aligned}
\int_{\pi / 4}^{\pi / 2} \varphi_{d}^{\prime}(\rho)^{2} \rho^{d-1} \mathrm{~d} \rho & =4 \int_{\pi / 4}^{\pi / 2} \frac{\varphi_{d}(2 \rho)^{2} \varphi_{d}^{\prime}(2 \rho)^{2}}{1-\varphi_{d}(2 \rho)^{2}} \rho^{d-1} \mathrm{~d} \rho \\
& =2^{2-d} \int_{\pi / 2}^{\pi} \frac{\varphi_{d}(\rho)^{2} \varphi_{d}^{\prime}(\rho)^{2}}{1-\varphi_{d}(\rho)^{2}} \rho^{d-1} \mathrm{~d} \rho
\end{aligned}
$$

so according to (15),

$$
V(\Phi) \propto \int_{\pi / 2}^{\pi} \varphi_{d}^{\prime}(\rho)^{2}\left(1+2^{2-d} \frac{\varphi_{d}(\rho)^{2}}{1-\varphi_{d}(\rho)^{2}}\right) \rho^{d-1} \mathrm{~d} \rho .
$$

\section{MinimiZATION}

We use techniques from the calculus of variations to reformulate the minimization problem. After making a simplifying substitution, we define the Euler-Lagrange equation for the stated problem; this is a differential equation that is satisfied by the minimizers of certain functionals [25]. The minimization is solved analytically, where the cases $d \leq 2$ and $d \geq 3$ are treated separately. The derived wavelet profiles $\varphi_{d}$ are plotted in Fig. 1, for $d \leq 4$.

The constant $k:=\sqrt{1-2^{2-d}}$, depending on $d$, appears several times in our calculations, so we define it here to simplify some expressions.
A. Case: $d=1,2$

We simplify (20) using the fact that $-1 \leq \varphi_{d} \leq 1$; i.e. we set $\varphi_{d}(\rho)=\cos (g(\rho))$. The resulting functional is

$$
V(\Phi) \propto \int_{\pi / 2}^{\pi} g^{\prime}(\rho)^{2}\left(1-k^{2} \cos (g(\rho))^{2}\right) \rho^{d-1} \mathrm{~d} \rho .
$$

Based on this representation, we define the functionals

$$
F(\rho, u, q):=q^{2}\left(1-k^{2} \cos (u)^{2}\right) \rho^{d-1}
$$

and

$$
I(g):=\int_{\pi / 2}^{\pi} F\left(\rho, g(\rho), g^{\prime}(\rho)\right) \mathrm{d} \rho
$$

so that $V(\Phi) \propto I(g)$. The boundary conditions for $g$ in the functional $I$ are $g(\pi / 2)=0$ and $g(\pi)=\pi / 2$.

The Euler-Lagrange equation for $I$ is

$$
0=\frac{\partial F}{\partial u}\left(\rho, g(\rho), g^{\prime}(\rho)\right)-\frac{\partial}{\partial \rho}\left(\frac{\partial F}{\partial q}\left(\rho, g(\rho), g^{\prime}(\rho)\right)\right),
$$

which is equivalent to

$$
\begin{aligned}
0= & k^{2} \rho \sin (2 g(\rho))\left(g^{\prime}(\rho)^{2}-2 g^{\prime}(\rho)\right) \\
& -2\left(1-k^{2} \cos (g(\rho))^{2}\right)\left(\rho g^{\prime \prime}(\rho)+(d-1) g^{\prime}(\rho)\right)
\end{aligned}
$$

For $d=1,(25)$ simplifies to

$0=\sin (2 g(\rho)) g^{\prime}(\rho)\left(g^{\prime}(\rho)-2\right)+2\left(1+\cos (g(\rho))^{2}\right) g^{\prime \prime}(\rho)$.

For $d=2,(25)$ simplifies to

$$
0=\rho g^{\prime \prime}(\rho)+g^{\prime}(\rho) .
$$

Proposition 3: For the cases $d=1$ and $d=2$, the unique $C^{2}$ minimizers of (23) are

$$
g(\rho)=\rho-\pi / 2
$$

and

$$
g(\rho)=\pi / 2 \log _{2}(2 \rho / \pi)
$$

respectively.

The wavelet profiles corresponding to the minimizers in Proposition 3 are

$$
\varphi_{1}(\rho)=\left\{\begin{array}{cc}
\cos (2 \rho-\pi) & \pi / 4 \leq \rho<\pi / 2 \\
\cos (\rho-\pi / 2) & \pi / 2 \leq \rho \leq \pi
\end{array}\right.
$$

and

$$
\varphi_{2}(\rho)=\cos \left(\frac{\pi}{2} \log _{2}\left(\frac{2 \rho}{\pi}\right)\right) .
$$

Notice that they are the Held and Simoncelli wavelets, which are depicted in Fig. 1.

\section{B. Case: $d \geq 3$}

For dimensions $d$ greater than 2, we use a different substitution. We make use of the Jacobi elliptic functions sn, cn and dn [26, Chapter 11]. They satisfy the addition formulas

$$
\operatorname{sn}(\rho ; k)^{2}+\operatorname{cn}(\rho ; k)^{2}=1
$$




$$
\operatorname{dn}(\rho ; k)^{2}+k^{2} \operatorname{sn}(\rho ; k)^{2}=1
$$

and the differentiation rules

$$
\begin{aligned}
& \frac{\partial \mathrm{sn}}{\partial \rho}(\rho ; k)=\operatorname{cn}(\rho ; k) \operatorname{dn}(\rho ; k) \\
& \frac{\partial \mathrm{dn}}{\partial \rho}(\rho ; k)=-k^{2} \operatorname{sn}(\rho ; k) \operatorname{cn}(\rho ; k) .
\end{aligned}
$$

We find a simplified form of (20) by making the substitution $\varphi_{d}=\operatorname{cn}(g(\rho) ; k)$. The result is

$$
V(\Phi) \propto \int_{\pi / 2}^{\pi} g^{\prime}(\rho)^{2} \operatorname{dn}(g(\rho) ; k)^{4} \rho^{d-1} \mathrm{~d} \rho .
$$

As in the case $d \leq 2$, we define functionals

$$
F(\rho, u, q):=q^{2} \operatorname{dn}(u ; k)^{4} \rho^{d-1}
$$

and

$$
I(g):=\int_{\pi / 2}^{\pi} F\left(\rho, g(\rho), g^{\prime}(\rho)\right) \mathrm{d} \rho
$$

so that $V(\Phi) \propto I(g)$. The boundary conditions for $g$ in the functional $I$ are

$$
\begin{aligned}
g(\pi / 2) & =\mathrm{cn}^{-1}(1 ; k)=0 \\
g(\pi) & =\mathrm{cn}^{-1}(0 ; k)=\int_{0}^{1} \frac{1}{\sqrt{\left(1-t^{2}\right)\left(1-k^{2} t^{2}\right)}} \mathrm{d} t .
\end{aligned}
$$

The Euler-Lagrange equation for $I$ is

$$
\begin{aligned}
0= & 2 k^{2} \rho \operatorname{sn}(g(\rho) ; k) \operatorname{cn}(g(\rho) ; k) g^{\prime}(\rho)^{2} \\
& -\operatorname{dn}(g(\rho) ; k)\left(\rho g^{\prime \prime}(\rho)+(d-1) g^{\prime}(\rho)\right) .
\end{aligned}
$$

Proposition 4: For $d \geq 3$, the unique $C^{2}$ minimizer of (36) is defined as follows. The value of $g(\rho)$ is the unique solution of the equation in $z$

$$
\frac{E(z, k)}{E(1, k)}=\frac{\pi^{d-2}-\rho^{d-2}}{2^{d-2} k^{2} \rho^{d-2}},
$$

where $E$ is the elliptic integral of the second kind

$$
E(z ; k):=\int_{0}^{z} \frac{\sqrt{1-k^{2} t^{2}}}{\sqrt{1-t^{2}}} \mathrm{~d} t .
$$

The resulting wavelet profiles $\varphi_{d}$ are plotted in Fig. 1, for $d=$ 3,4 .

\section{Summary AND Future WORK}

We have considered the problem of identifying the optimal band-limited isotropic mother wavelet for a tight frame, using a localization criterion. We found analytic solutions for arbitrary spatial dimension $d$. In the cases $d=1$ and $d=2$, we obtained previously proposed wavelet profiles, namely Held $(d=1)$ and Simoncelli $(d=2)$.

In this work, we focused on the localization criterion with parameter $n=1$, and the selection of optimal wavelets for $n>1$ remains open. We intend to address this point in the future.

\section{REFERENCES}

[1] J. R. Romero, S. K. Alexander, S. Baid, S. Jain, and M. Papadakis, "The geometry and the analytic properties of isotropic multiresolution analysis," Adv. Comput. Math., vol. 31, no. 1-3, pp. 283-328, 2009.

[2] I. Daubechies, B. Han, A. Ron, and Z. Shen, "Framelets: MRA-based constructions of wavelet frames," Appl. Comput. Harmon. Anal., vol. 14, no. 1, pp. 1-46, 2003.

[3] M. Unser, N. Chenouard, and D. Van De Ville, "Steerable pyramids and tight wavelet frames in $L_{2}\left(\mathbf{R}^{d}\right)$," IEEE Trans. Image Process., vol. 20, no. 10, pp. 2705-2721, 2011.

[4] I. Daubechies, A. Grossmann, and Y. Meyer, "Painless nonorthogonal expansions," J. Math. Phys., vol. 27, no. 5, 1986.

[5] S. Held, M. Storath, P. Massopust, and B. Forster, "Steerable wavelet frames based on the riesz transform," IEEE Trans. Image Process., vol. 19, no. 3, pp. 653-667, 2010.

[6] J. Portilla and E. P. Simoncelli, "A parametric texture model based on joint statistics of complex wavelet coefficients," Int. J. Comput. Vis., vol. 40, pp. 49-70, 2000.

[7] N. Chenouard and M. Unser, "3D steerable wavelets in practice," IEEE Trans. Image Process., vol. 21, no. 11, pp. 4522-4533, Nov. 2012.

[8] A. Depeursinge, D. Van de Ville, A. Platon, A. Geissbuhler, P.-A. Poletti, and H. Muller, "Near-affine-invariant texture learning for lung tissue analysis using isotropic wavelet frames," IEEE Trans. Inf. Technol. Biomed., vol. 16, no. 4, pp. 665-675, 2012.

[9] M. Unser and N. Chenouard, "A unifying parametric framework for 2D steerable wavelet transforms," SIAM J. Imag. Sci., vol. 6, no. 1, pp. 102-135, 2013.

[10] E. Bostan, M. Unser, and J. P. Ward, "Divergence-free wavelet frames," IEEE Signal Process. Lett., vol. 22, no. 8, pp. 1142-1145, Aug. 2015.

[11] D. Gabor, "Theory of communication. part 1: The analysis of information," J. Inst. Electr. Eng. III: Radio Commun. Eng., vol. 93, no. 26, pp. 429-441, 1946.

[12] D. Van De Ville, T. Blu, and M. Unser, "Isotropic polyharmonic B-splines: Scaling functions and wavelets," IEEE Trans. Image Process., vol. 14, no. 11, pp. 1798-1813, 2005.

[13] D. Slepian and H. O. Pollak, "Prolate spheroidal wave functions, fourier analysis and uncertainty-I," Bell Syst. Tech. J., vol. 40, pp. 43-63, 1961.

[14] H. J. Landau and H. O. Pollak, "Prolate spheroidal wave functions, fourier analysis and uncertainty-II," Bell Syst. Tech. J., vol. 40, pp. 65-84, 1961.

[15] H. J. Landau and H. O. Pollak, "Prolate spheroidal wave functions, fourier analysis and uncertainty-III. The dimension of the space of essentially time- and band-limited signals," Bell Syst. Tech. J., vol. 41, pp. 1295-1336, 1962.

[16] D. Slepian, "Prolate spheroidal wave functions, fourier analysis and uncertainity-IV. Extensions to many dimensions; generalized prolate spheroidal functions," Bell Syst. Tech. J., vol. 43, pp. 3009-3057, 1964.

[17] D. Slepian, "Prolate spheroidal wave functions, fourier analysis, and uncertainty-V: The discrete case," Bell Syst. Tech. J., vol. 57, no. 5, pp. 1371-1430, 1978.

[18] C. K. Chui, J. Stöckler, and J. D. Ward, "Analytic wavelets generated by radial functions," Adv. Comput. Math., vol. 5, no. 1, pp. 95-123, 1996.

[19] F. J. Narcowich, P. Petrushev, and J. D. Ward, "Localized tight frames on spheres," SIAM J. Math. Anal., vol. 38, no. 2, pp. 574-594, 2006.

[20] F. J. Narcowich and J. D. Ward, "Nonstationary wavelets on the $m$-sphere for scattered data," Appl. Comput. Harmon. Anal., vol. 3, no. 4, pp. 324-336, 1996.

[21] P. Pad, V. Uhlmann, and M. Unser, "VOW: Variance-optimal wavelets for the steerable pyramid," in IEEE Int. Conf. Image Processing (ICIP), Oct. 2014, pp. 2973-2977.

[22] H. Wendland, Scattered Data Approximation, ser. Cambridge Monogr. Appl. Comput. Math.. Cambridge, U.K.: Cambridge Univ. Press, 2005, vol. 17.

[23] R. Schaback, "The missing wendland functions," Adv. Comput. Math., vol. 34 , no. 1 , pp. $67-81,2011$.

[24] I. Daubechies, "Ten lectures on wavelets," SIAM, vol. 61, 1992.

[25] J. Jost and X. Li-Jost, Calculus of variations, ser. Cambridge Studies in Advanced Mathematics. Cambridge, U.K.: Cambridge Univ. Press, 1998, vol. 64 .

[26] R. Beals and R. Wong, Special functions, ser. Cambridge Studies in Advanced Mathematics. Cambridge, U.K.: Cambridge Univ. Press, 2010, vol. 126. 